\title{
iTRAQ-based quantitative proteomic analysis of cervical cancer
}

\author{
YIBING DING ${ }^{1,2}$, MIN YANG $^{3}$, SHA SHE $^{3}$, HAIYAN MIN $^{1,2}$, XIAOMING XV $^{3}$, XIAOPING RAN $^{3}$, \\ YONGZHENG WU ${ }^{1,2}$, WEI WANG ${ }^{1,2}$, LEI WANG ${ }^{1,2}$, LONG YI $^{1,2}$, YIXUAN YANG ${ }^{3}$ and QIAN GAO $^{1,2}$ \\ ${ }^{1}$ Center for Translational Medicine, ${ }^{2}$ Jiangsu Key Laboratory for Molecular Medicine, \\ Nanjing University Medical School, Nanjing, P.R. China; ${ }^{3}$ Department of Infectious Diseases, \\ Second Affiliated Hospital of Chongqing Medical University, Chongqing, P.R. China
}

Received November 13, 2014; Accepted December 29, 2014

DOI: 10.3892/ijo.2015.2859

\begin{abstract}
Cervical cancer is the seventh most common cancer overall and the third among females. To obtain systematic insight into the protein profile that participates in cervical tumor oncogenesis and improve the current target therapies, iTRAQ labeling and NanoLC-MS/MS analysis were utilized to detect differentially expressed proteins in cervical cancer. As a result, 3,647 proteins were identified, among which the expression levels of 294 proteins in cervical cancer samples were distinct from the paired non-tumor samples. Further validation of the differentially expressed proteins, including G6PD, ALDH3A1, STAT1 and HSPB1, was carried out via qRT-PCR, western blot analysis and tissue microarray. Functional analysis of one of the highly expressed proteins, G6PD, was performed using RNA interference. Attenuated G6PD expression reduced the capacity of HeLa cells to migrate and invade in vitro. Our investigation complemented the understanding of cervical cancer progression. Furthermore, the present study supports the notion that suppressing the expression of G6PD may be a promising strategy in developing novel cancer therapeutic drugs.
\end{abstract}

\section{Introduction}

Cervical tumor is the seventh most common cancer overall and the third among women (1). Annually, more than 500,000 new cases of invasive cervical carcinoma are diagnosed globally, representing $10 \%$ of all cancers in females. Furthermore, $80 \%$ of the cases occur in developing countries and the survival rate is still poor (2). Although significant advances

Correspondence to: Professor Qian Gao, Center for Translational Medicine, Nanjing University Medical School, 22 Hankou Road, Nanjing, Jiangsu 210093, P.R. China

E-mail: qian_gao@nju.edu.cn

Dr Yixuan Yang, Department of Infectious Diseases, Second Affiliated Hospital of Chongqing Medical University, 74 Linjiang Road, Chongqing 400010, P.R. China

E-mail: yixuan.yang@hotmail.com

Key words: cervical cancer, G6PD, iTRAQ, proteomics, RNAi have been made in cervical cancer diagnosis, late stage diagnosis and poor survival are major challenges. As infection by human papillomavirus (HPV) types 16 and 18 has been shown to be a central causal agent for cervical cancer $(3,4)$, consensus panels of the International Agency for Research on Cancer (IARC) and WHO concluded that there is enough justification to evaluate HPV testing as an adjunct to Papanikolaou (Pap) smear screening for cervical cancer $(5,6)$. However, due to the lack of knowledge regarding the development and progression of cervical cancer, it still has one of the highest mortality rates among the common malignancies in women. It is urgent to provide insight into the mechanism of cervical cancer, with the expectation of finding more effective and alternative molecular targets for treatment of this disease.

Comparative proteomic analysis provides a comprehensive evaluation of protein expression, and has been widely applied in cancer research (7). For example, Annexin A2 (ANXA2) was demonstrated as a factor linked to cell transformation and oncogenesis in cervical cancer via two-dimensional electrophoresis (2-DE) and MALDI-TOF mass spectrometric analysis (8). Based on 2-DE analysis between human cervical squamous cell carcinoma (SCC) and normal cervical tissues, overexpression of cytokeratin-19 was linked to prosoplasia and uncontrolled proliferation of cervical epithelial tissue (9). In another investigation, differences in the abundance of zinca-2-glycoprotein (ZAG) in serum between endometrial adenocarcinoma patients and cervical tumor patients were examined by $2-\mathrm{DE}$ with silver staining. Moreover, ZAG was identified as an indicator linked to the development of cachexia (10). However, considerable inter-gel variation, low sensitivity and excessive time/labor costs are common disadvantages with the standard 2-DE approach $(7,11)$.

Isotope-based quantitative proteomics have been employed in analyzing post-translational modifications or discovering biomarkers. Compared to other isotope-based technologies, such as isotope-coded affinity tags (ICAT), 180 and stable isotope labeling with amino acids in cell culture (SILAC) (12-14), the isobaric tags for relative and absolute quantitation (iTRAQ) method is deemed to be outstanding, since it can analyze multiple samples in one experiment for simultaneous analysis. In the present study, iTRAQ labeling coupled with high resolution mass spectrometry was carried out to detect the difference in proteins between cervical cancer and non-cancer samples. We expected to find host proteins 
Table I. Experimental procedures of high performance liquid chromatography (HPLC) for peptide fractionation and NanoLC-MS/MS analysis.

\begin{tabular}{|c|c|c|c|c|c|}
\hline \multicolumn{3}{|c|}{ RP-HPLC for peptide fractionation } & \multicolumn{3}{|c|}{ NanoLC-MS/MS analysis } \\
\hline $\begin{array}{l}\text { Gradient } \\
\text { elution }\end{array}$ & $\begin{array}{l}\text { Mobile phase A } \\
\text { (20 mM ammonium } \\
\text { formate, } \mathrm{pH} 10.0)\end{array}$ & $\begin{array}{c}\text { Mobile phase B } \\
\text { (80\% acetonitrile with } 20 \mathrm{mM} \\
\text { ammonium formate, } \mathrm{pH} 10.0 \text { ) }\end{array}$ & $\begin{array}{c}\text { Gradient } \\
\text { elution }\end{array}$ & $\begin{array}{c}\text { Mobile phase } \mathrm{A} \\
\text { (2\% acetonitrile with } \\
0.1 \% \text { formic acid) }\end{array}$ & $\begin{array}{c}\text { Mobile phase } \mathrm{B} \\
\text { (98\% acetonitrile with } \\
0.1 \% \text { formic acid) }\end{array}$ \\
\hline $0 \mathrm{~min}$ & $95 \%$ & $5 \%$ & $0 \mathrm{~min}$ & $95 \%$ & $5 \%$ \\
\hline $5 \mathrm{~min}$ & $95 \%$ & $5 \%$ & $5 \mathrm{~min}$ & $95 \%$ & $5 \%$ \\
\hline $25 \mathrm{~min}$ & $77 \%$ & $23 \%$ & $85 \mathrm{~min}$ & $75 \%$ & $25 \%$ \\
\hline $40 \mathrm{~min}$ & $55 \%$ & $45 \%$ & $105 \mathrm{~min}$ & $50 \%$ & $50 \%$ \\
\hline $41 \mathrm{~min}$ & $10 \%$ & $90 \%$ & 106 min & $20 \%$ & $80 \%$ \\
\hline $45 \mathrm{~min}$ & $10 \%$ & $90 \%$ & $110 \mathrm{~min}$ & $20 \%$ & $80 \%$ \\
\hline $46 \min$ & $95 \%$ & $5 \%$ & $111 \mathrm{~min}$ & $95 \%$ & $5 \%$ \\
\hline $60 \min$ & $95 \%$ & $5 \%$ & $120 \mathrm{~min}$ & $95 \%$ & $5 \%$ \\
\hline \multicolumn{3}{|c|}{ Detector: UV 220 nm } & \multicolumn{3}{|c|}{ Detector: Triple-TOF 5600 MS/MS } \\
\hline
\end{tabular}

RP-HPLC for peptide fractionation: column, Gemini-NX C18 (4.6 mm x 250 mm, $5 \mu \mathrm{m} 110$ A; Phenomenex, Torrance, CA, USA); flow rate: $800 \mu \mathrm{l} / \mathrm{min}$. NanoLC-MS/MS analysis: column, C18 trap ( $5 \mu \mathrm{m}, 0.3 \mathrm{~mm}$ x $5 \mathrm{~mm}$; Agilent Technologies, Palo Alto, CA, USA); house-packed NanoLC C18 (200 ̊, $5 \mu \mathrm{m}, 75 \mu \mathrm{m} \times 10 \mathrm{~cm})$; flow rate, $300 \mathrm{nl} / \mathrm{min}$.

associated with cervical cancer that could be potential targets for diagnosis or future treatment regimens.

\section{Materials and methods}

Tissues and cell line. The present study was approved by the Institutional Review Board for Human Subject Review. Written informed consent was obtained from all the patients in accordance with the revised Helsinki Declaration. A total of 8 human cervical cancer samples and 8 paired non-cancer samples were collected at the Second Affiliated Hospital of Chongqing Medical University. The HeLa cell line was purchased from the Cancer Research Department of China Medical Science Institute.

iTRAQ labeling and peptide fractionation. Total proteins $(100 \mu \mathrm{g})$ extracted from each sample were denatured, cysteine blocked, and digested as described in the standard protocol of the iTRAQ kit (AB Sciex, Framingham, MA, USA). Pooled non-tumor samples were labeled with iTRAQ tags 113 and 115, and tags 114 and 116 were used to label pooled cervical tumor samples. The labeled peptides were pooled in 1:1:1:1 ratio and lyophilized. The mixture was separated with an LC-30 high performance liquid chromatography system (Shimadzu, Kyoto, Japan) by gradient elution. The detailed procedures are shown in Table I. Ten fractions were collected and lyophilized for further analysis.

NanoLC-2D-TripleTOF 5600 analysis. A Triple TOF 5600 system coupled to an Eksigent NanoLC-2D system (AB Sciex) was used for protein identification and quantization. Each fraction was separated in a 2-h gradient elution by the NanoLC-2D system (Table I). The mass spectrometer was set in the positive ion mode at a mass range of $350-1,500 \mathrm{~m} / \mathrm{z}$, with a 0.25 -sec accumulation time, followed by information-dependent acqui- sition (IDA). The top 30 precursor ions within each cycle were automatically selected for fragmentation, with each MS/MS spectrum accumulated for $0.1 \mathrm{sec}(100-1,500 \mathrm{~m} / \mathrm{z})$.

ProteinPilot v.4.5 software (AB Sciex) was used for data search against the UniProt database. The standard searching parameters and false discovery rate analysis were set. A threshold of confidence $>99 \%$ and a local false discovery rate (FDR) of $<1 \%$ were used for both protein identification and quantitative analysis. More than 2 unique peptides were required for protein identification. P-values $<0.01$ were required for relative quantification. The PeakView 1.1 software was used to extract ion chromatograms.

Quantitative real time-PCR analysis ( $q R T-P C R$ ). Total RNA was extracted using the RNeasy Mini kit (Qiagen, Hilden, Germany), and $100 \mathrm{ng}$ of total RNA was reverse transcribed into cDNA using the PrimeScript RT reagent kit (Takara, Dalian, China). qRT-PCR assays were performed on an ABI 7900HT system with TaqMan kits. Primers for G6PD (Hs00166169-m1), STAT1 (Hs01014007_m1), HSPB1 (Hs03044127_g1), DCN (Hs00466796_CE), ALDH3A1 (Hs00964880_m1), EPX (Hs00417510_CE), PRG3 (Hs00196082_m1), OGN (Hs00247901_m1), CRNN (Hs00211833_m1), AGR2 (Hs00356521_m1), ORM2 (Hs00301996_CE) and GAPDH (Hs02758991_g1) were used. Each sample was run in triplicate, and all reactions were performed at least twice. The $2^{-\Delta \Delta \mathrm{CT}}$ method was used for data analysis.

Western blot analysis. Total protein $(20 \mu \mathrm{g})$ extracted from each tissue sample was separated by SDS-PAGE, and transferred to polyvinylpyrrolidone membrane (Amersham Biosciences, Uppsala, Sweden). Primary antibodies (1:500-1:1,000) against G6PD, STAT1, HSPB1, ALDH3A1 and FSCN1 (Abcam, Cambridge, MA, USA) and HRP-conjugated secondary antibodies (1:5,000; Amersham Biosciences) were used to incubate 
A

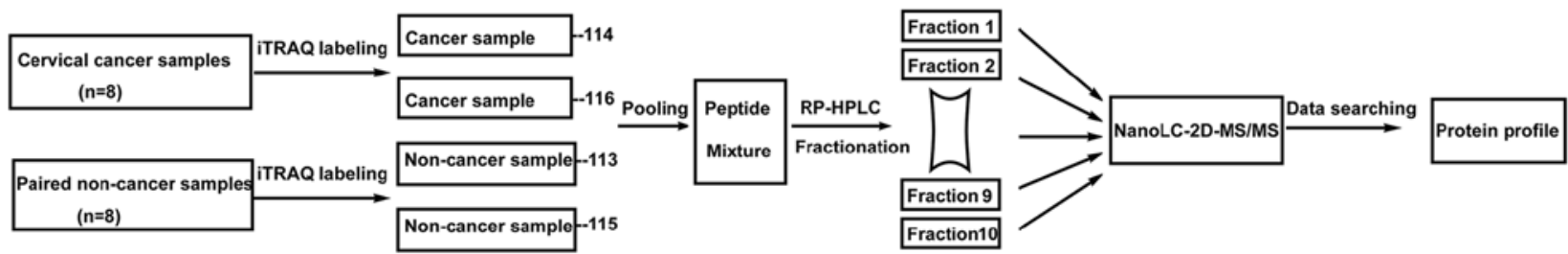

B

B LFYLALPPTVYEAVTK

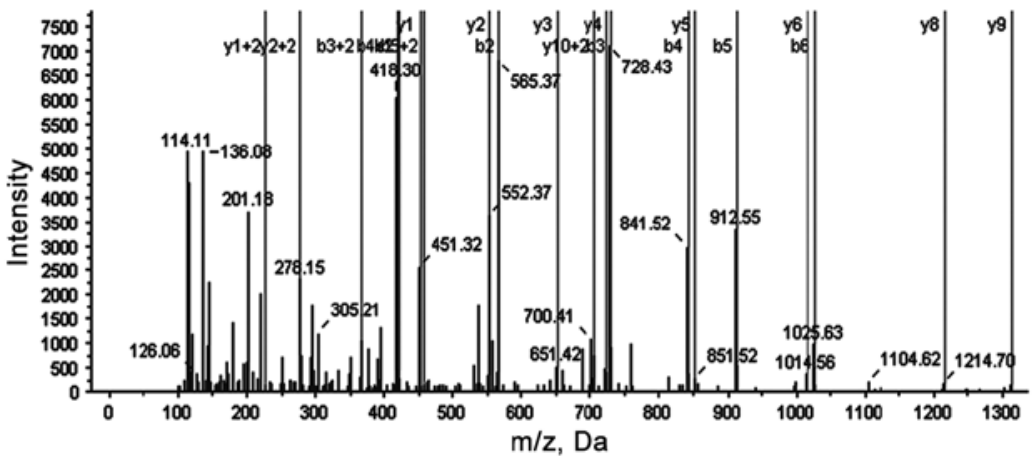

C

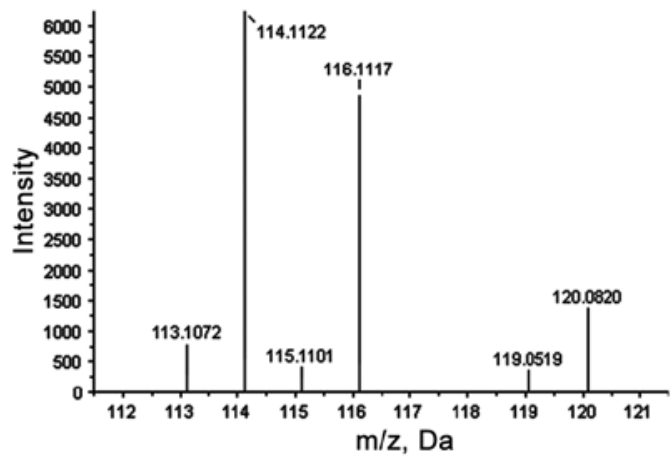

Figure 1. The iTRAQ-based proteomics analysis for protein profiling in cervical cancer. (A) Schematic workflow. (B) Representative MS/MS spectrum of a peptide with 16 amino acids (LFYLALPPTVYEAVTK) from G6PD. (C) The ratio of tags 114:113 and 116:115 indicated the relative protein level of G6PD in pooled cervical cancer samples compared with that in pooled non-cancer samples.

the samples. Bands were detected with an ECL detection system (Amersham Biosciences). Each sample was analyzed at least twice. Detailed procedure of experiments was described in a previous study (15).

Immunohistochemistry (IHC) and tissue microarray. The tissue microarrays (CR802) were purchased from US Biomax, Inc. (Rockville, MD, USA) to detect G6PD, STAT1, HSPB1 and ALDH3A1 in cores from 40 cervical tumor tissues and 40 non-tumor tissues, antibodies against G6PD (1:90), STAT1 (1:200), HSPB1 (1:250) and ALDH3A1 (1:200) were added and incubated overnight at $4^{\circ} \mathrm{C}$. Detection was performed with the Envision/horseradish peroxidase system (DakoCytomation, Glostrup, Denmark). Semi-quantification of protein expression was defined by scoring criteria. The positive cells (\%) and staining intensity (scale $0-3$ ) were checked, which were then multiplied to yield a score ranging from 0 to 300 . To maintain consistency, the same qualified pathologist gave interpretations for all IHC data.

Wound healing and invasion assay. G6PD-specific (50 nM) siRNAHSS103891, HSS103892 and HSS103893 (Invitrogen, Carlsbad, CA, USA) or negative control siRNA (12935-400; Invitrogen) were transfected into HeLa cells $\left(10^{5}\right.$ cell/well $)$ After $48 \mathrm{~h}$, wound healing and invasion experiments were performed on 6-well plates seeded with HeLa cells. After the cells reached confluency, a $200 \mu \mathrm{l}$ pipette tip was used to incise the cell monolayer. The debris was rinsed away and removed. The extent of gap closure was monitored and photographed under a microscope up to $24 \mathrm{~h}$. The invasion assays were performed using a Cell Invasion Assay kit (Cell Biolabs, San Diego, CA, USA), following the manufacturer's instructions. After $24 \mathrm{~h}$, the number of cells that invaded and attached to the bottom chamber was measured by CyQuant GR fluorescent dye $(560 \mathrm{~nm})$.
Statistical analyses. Statistical analyses were performed by SPSS software v13.0 using the Student'st-test, Mann-Whitney U-test, $\chi^{2}$ test or Spearman's rank correlation analysis. A P-value $<0.05$ was considered statistically significant. All tests of significance were two-tailed.

\section{Results}

Functional profiles of differentially expressed proteins in cervical tumors. In total, 3,647 proteins were identified with $1 \%$ global FDR from fit in cervical cancer following a workflow shown in Fig. 1. For subsequent relative quantification analysis, a cut-off of 1.3-fold change, up or down, was applied to all iTRAQ ratios to minimize false positives when identifying proteins as overerexpressed or downregulated. This process is widely adopted in other proteomics investigations (16-19). Accordingly, 294 proteins were identified as differentially expressed in pooled cervical tumor tissues comparing to non-tumor tissues, including 130 upregulated and 164 downregulated proteins. The top 30 upregulated proteins and top 30 downregulated proteins were listed in Table II.

To obtain the functional characteristics of proteins associated with cervical carcinoma oncogenesis, we classified the 294 proteins using Protein Analysis through Evolutionary Relationships Classification System (PANTHER, www. pantherdb.org). Twelve biological processes are involved, with $55.0 \%$ of the proteins participating in metabolic processes, followed by cellular processes $(37.1 \%)$ and developmental processes (19.4\%). According to molecular function, the proteins were divided into 10 categories, including catalytic activity (37.8\%), binding activity (32.0\%) and structural molecule activity (19.4\%). The 294 proteins were grouped into 27 protein classes, including cytoskeletal protein (14.7\%), hydrolase (12.9\%) and nucleic acid binding proteins (10.4\%). A total of 63 signaling pathways were associated, with inte- 


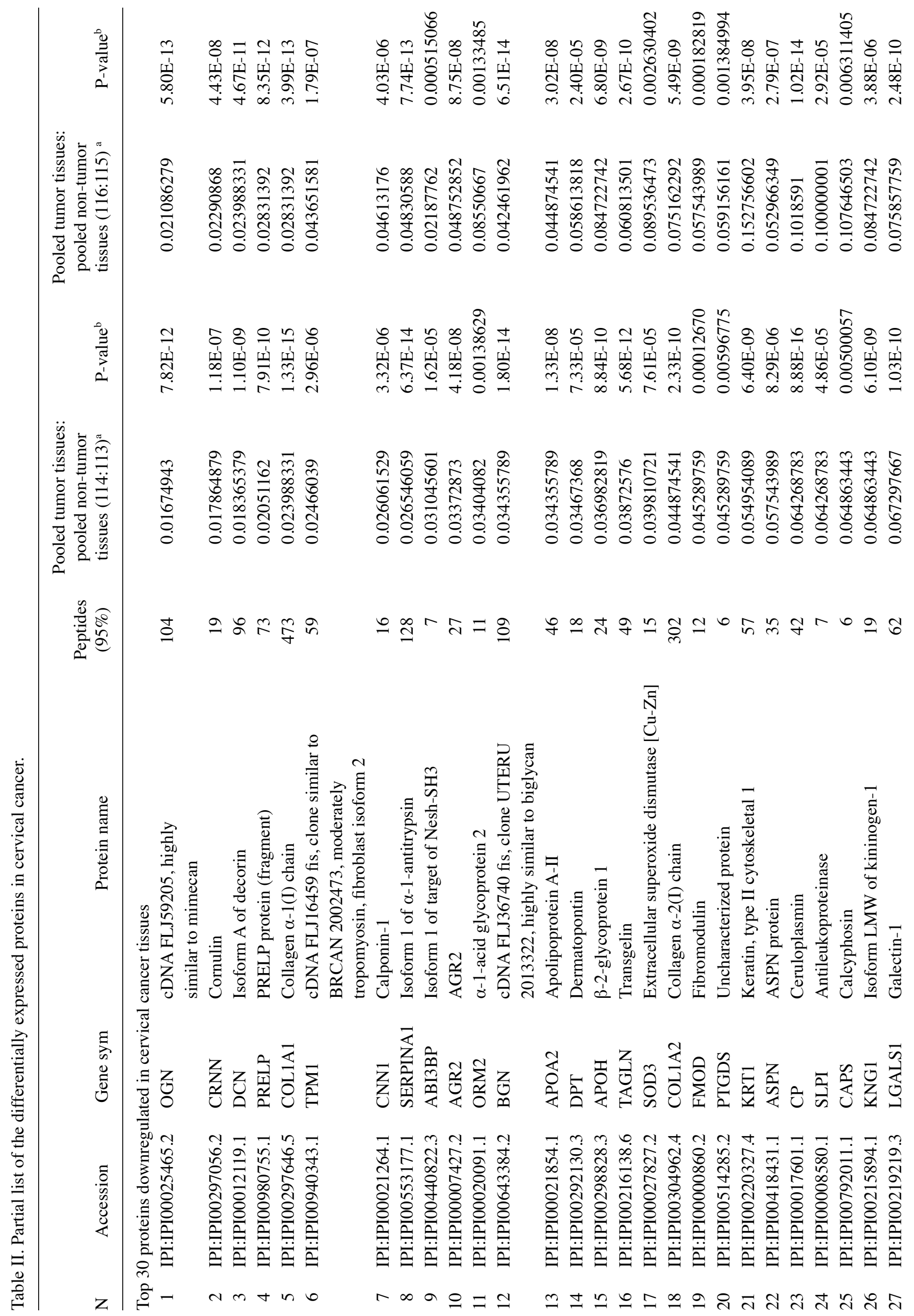




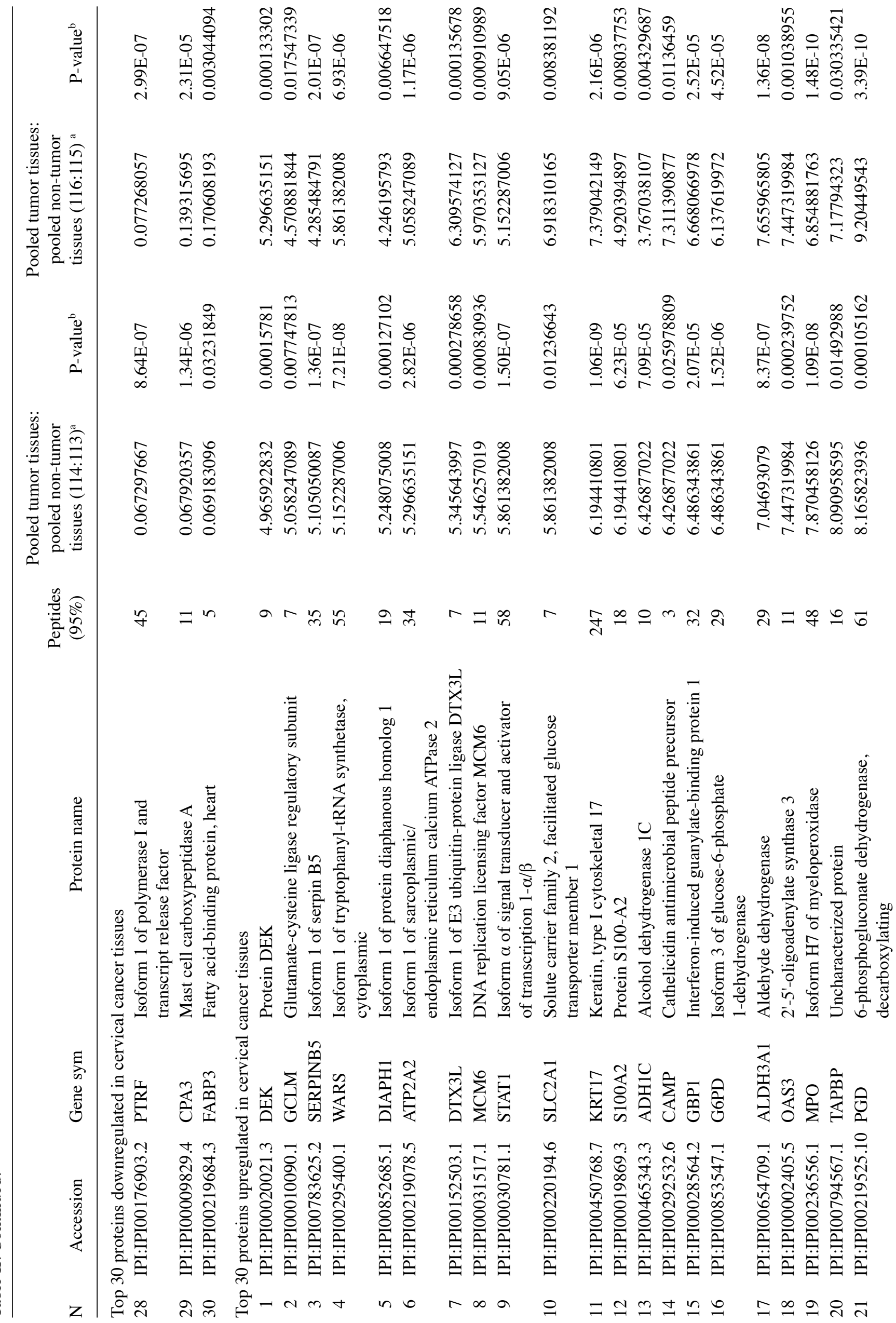


grin signaling pathway (3.2\%), blood coagulation (2.9\%) and inflammation mediated by chemokine and cytokine signaling pathway (2.2\%) at the top of the list (Fig. 2).

Validation of altered expression levels of proteins. The iTRAQ study results were further validated by qRT-PCR and western blot analyses. Fig. 3A shows the relative mRNA levels of selected differentially expressed proteins in the cervical tumor tissues, compared to those in the paired non-tumor tissues. The mRNA levels of G6PD, HSPB1, STAT1, ALDH3A1, FSCN1, EPX and PRG3 were found to be upregulated, whereas the levels of DCN, OGN, CRNN, AGR2 and ORM2 were downregulated. The upregulation of protein levels of G6PD, HSPB1, STAT1, ALDH3A1 and FSCN1 were subsequently detected by western blot analysis (Fig. 3B). This trend matched that observed in the iTRAQ method.

Expression of G6PD, HSPB1, STAT1 and ALDH3A1 in tissue array. The clinical relevance of G6PD, HSPB1, STAT1 and ALDH3A1 in cervical cancer was assessed by IHC analysis (Fig. 4). A tissue microarray including 40 cervical cancer tissues and 40 matched or unmatched non-cancer cervical tissues was analyzed. As a result, cervical cancer samples showed significantly higher levels of G6PD, HSPB1, STAT1 and ALDH3A1 than those in controls. Moreover, G6PD expression was detected in $100 \%$ (40/40) of cervical cancer samples, compared to $45 \%$ (18/40) in controls. The staining intensity of G6PD in cervical cancer cells was much stronger than that in control epithelial cells. Similar trends were observed in the IHC analysis of HSPB1, STAT1 and ALDH3A1.

G6PD knockdown compromised HeLa cells' invasion and migration. The dramatic increase of G6PD in cervical cancer suggested that G6PD not only contributes to the biosynthesis of cervical cancer cells, but also is crucial for their malignancy. To test this hypothesis, HeLa, a human cervical cancer cell line, was tested with an RNA interference assay. G6PD expression in HeLa cells was initially silenced by transfection of G6PD-specific siRNAs (Fig. 5A). G6PD-silenced HeLa cells and control cells were then subjected to invasion and migration assays. Invasion capacity of G6PD-silenced cells was inhibited by $30-40 \%$ when compared to that of the control cells $(\mathrm{P}<0.01)$ (Fig. 5B). The readout of the scratch wound repair assays was reduced by $55-65 \%$ in G6PD-silenced cells when compared to that of the control cells $(\mathrm{P}<0.01)$ (Fig. 5C). Our results supported the notion that G6PD may be an effective target in cervical cancer treatment.

\section{Discussion}

Cervical cancer remains one of the leading causes of death among women throughout the world. The morbidity and death rates remain frustratingly high in spite of several emerging biomarkers for diagnosis. Discovery of novel factors that are related to the biology of cervical carcinoma is an urgent task for improving current treatment strategies and prognosis of the disease.

In the present study, we identified proteins differentially expressed between tumor and non-tumor cervical tissues via the iTRAQ proteomics approach. Verification studies using 


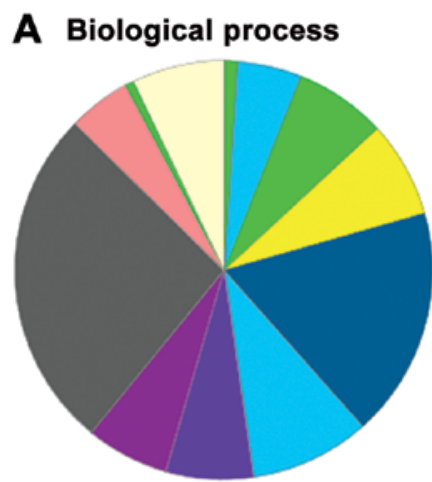

B Go molecular function

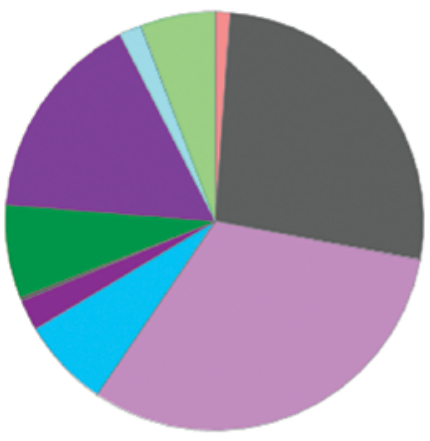

C Panther protein class

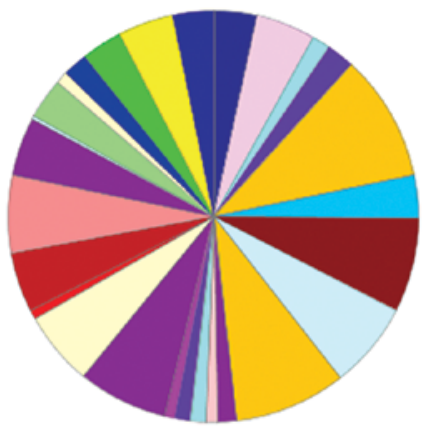

apoptotic process (GO:0006915)

| biological adhesion (GO:0022610)

biological regulation (GO:0065007)

cellular component organization or biogenesis (GO:0071840)

cellular process (GO:0009987)

developmental process (GO:0032502)

immune system process (GO:0002376)

localization (GO:0051179)

metabolic process (GO:0008152)

multicellular organismal process (GO:0032501)

reproduction (GO:0000003)

response to stimulus (GO:0050896)

antioxidant activity (GO:0016209)

binding (GO:0005488)

catalytic activity (GO:0003824)

enzyme regulator activity (GO:0030234)

nucleic acid binding transcription factor activity (GO:0001071)

protein binding transcription factor activity (GO:0000988)

receptor activity (GO:0004872)

structural molecule activity (GO:0005198)

translation regulator activity (GO:0045182)

transporter activity (GO:0005215)

calcium-binding protein (PC00060)

cell adhesion molecule (PC00069)

cell junction protein (PC00070)

chaperone (PC00072)

cytoskeletal protein (PC00085)

defense/immunity protein (PC00090)

enzyme modulator (PC00095)

extracellular matrix protein (PC00102)

hydrolase (PC00121)

isomerase (PC00135)

kinase (PC00137)

ligase (PC00142)

lyase (PC00144)

membrane traffic protein (PC00150) nucleic acid binding (PC00171) oxidoreductase (PC00176)

phosphatase (PC00181)

protease (PC00190)

receptor (PC00197)

signaling molecule (PC00207)

storage protein (PC00210)

structural protein (PC00211)

surfactant (PC00212)

transcription factor (PC00218)

transfer/carrier protein (PC00219)

transferase (PC00220)

transporter (PC00227)

Figure 2. Classification of 294 differentially expressed proteins in cervical cancer according to (A) biological process, (B) molecular function and (C) molecular protein class. Data were analyzed using the Protein Analysis through Evolutionary Relationships Classification System (PANTHER, www.pantherdb.org/).

qRT-PCR, immunoblot and IHC assays confirmed that the expressions of G6PD, STAT1, ALDH3A1 and HSPB1 were, indeed, significantly increased in tumor tissues. Functional studies indicated that G6PD tends to act on migration and invasion of cervical cancer cells. Our findings revealed that the iTRAQ method for large-scale protein quantification is amenable to high throughput processes and credible, and some novel proteins uncovered here may serve as potential targets for cervical tumor treatment.

G6PD, as the first and rate-limiting enzyme in the ubiquitous pentose phosphate pathway (PPP) (20), participates in biological processes of various cancers (21-23). Through PPP, the biosynthesis and rapid proliferation of tumor cells are supported $(24,25)$. In non-tumor cells, G6PD binds with p53 protein to prevent it from forming the active dimer (26). This process, however, inhibits PPP by quenching functional G6PD (27). Notably, this inhibition of G6PD by p53 does not exist in tumor cells, because mutant p53 loses its inhibitory effect on G6PD, and, thus, results in elevated PPP glucose flux, which ultimately enhances glucose consumption and promotes tumor cell biosynthesis (26). In other words, G6PD motivates cancer cell proliferation and contributes to tumorigenesis by increasing PPP glucose flux. Since the relationship between G6PD and cervical cancer was not well investigated previously, we examined the effect of G6PD proteins on HeLa cells. As a consequence, the invasion and migration capabilities of 


\section{A}

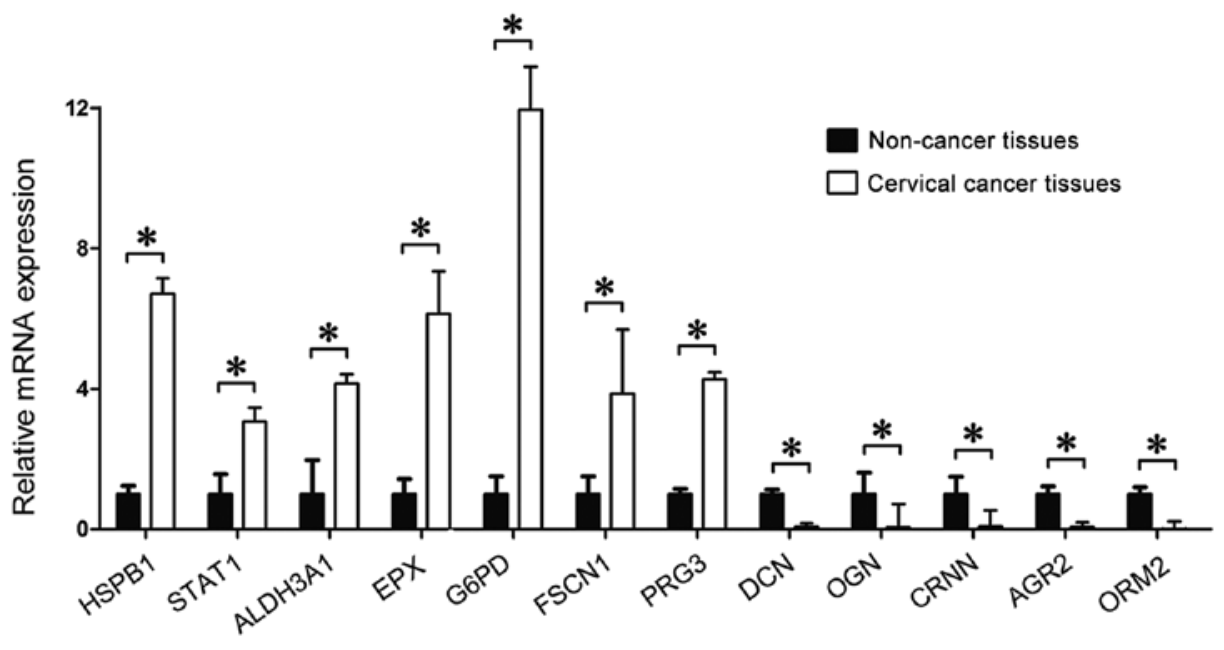

\section{B}

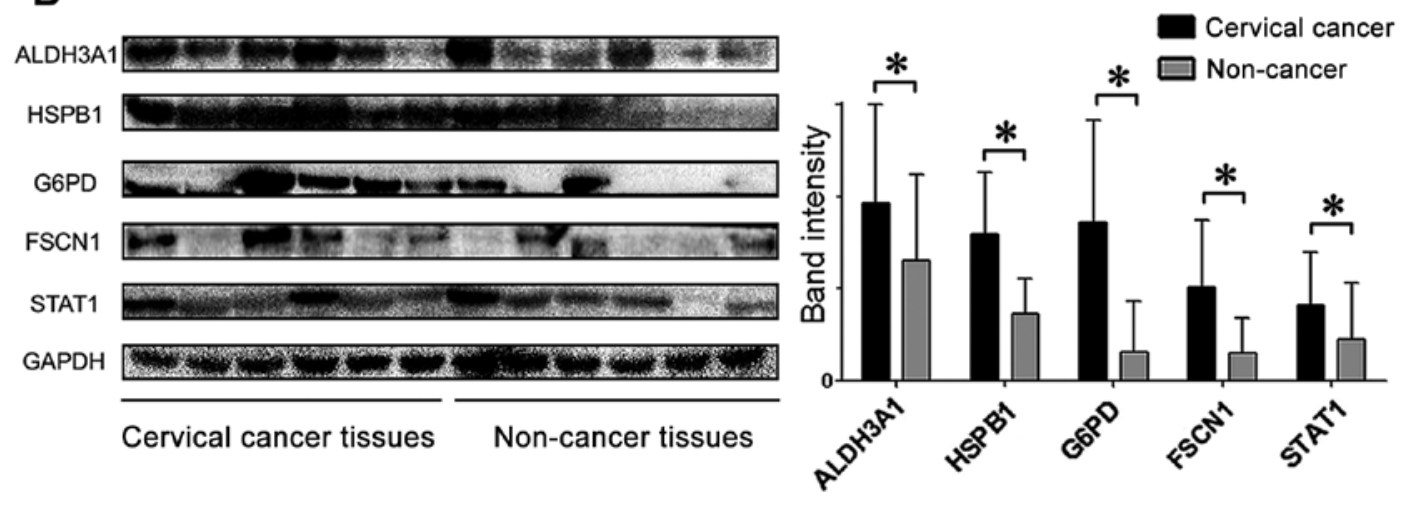

Figure 3. qRT-PCR and western blot analyses of differentially expressed proteins in cervical cancer. The relative mRNA expression levels of HSPB1, STAT1, ALDH3A1, EPX, G6PD, FSCN1, PRG3, DCN, OGN, CRNN, AGR2 and ORM2 (A), and the high protein levels of ALDH3A1, HSPB1, G6PD, FSCN1 and STAT1 (B) in cervical cancer tissues compared with non-cancer cervical tissues. Bars indicate SD. ${ }^{*} \mathrm{P}<0.05$.

A IHC:ALDH3A1

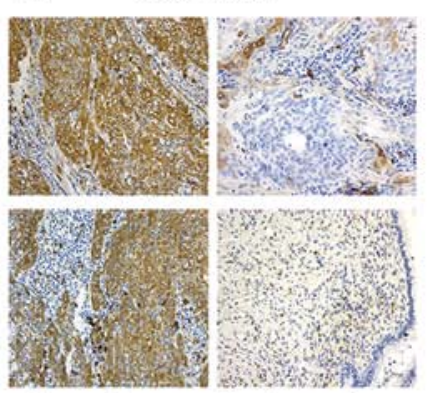

Tumor tissues Non-tumor tissues

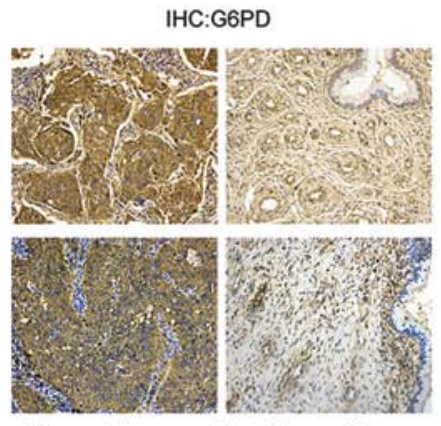

Tumor tissues Non-tumor tissues

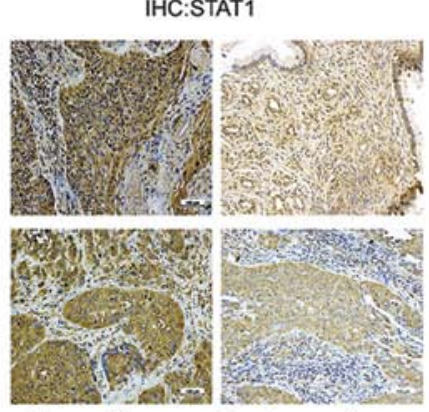

Tumor tissues Non-tumor tissues

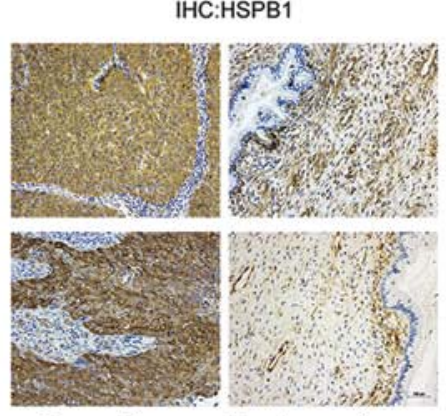

Tumor tissues Non-tumor tissues

B
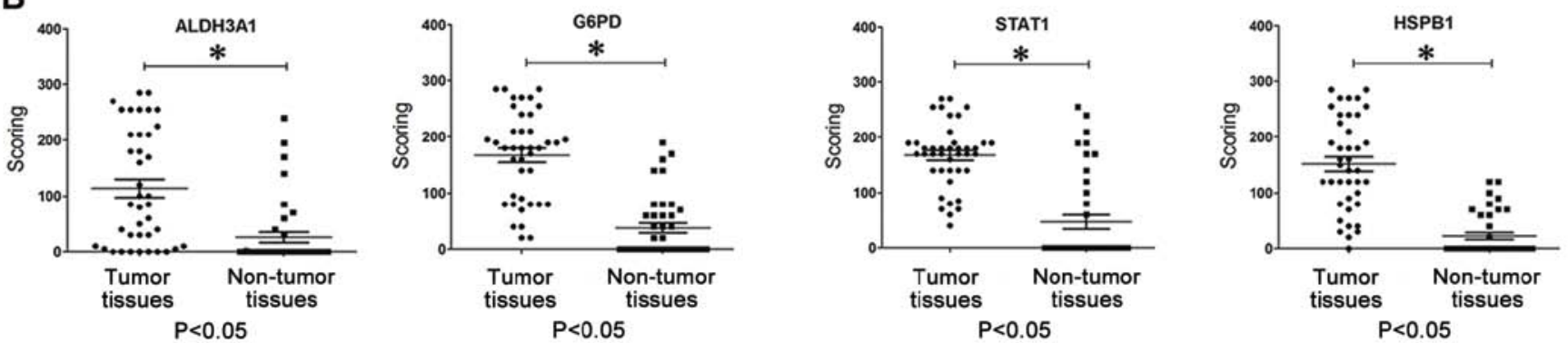

Figure 4. Representative IHC images of ALDH3A1, G6PD, STAT1 and HSPB1 in tissue microarrays of 40 cervical cancer tissues and 40 matched or unmatched non-cancer cervical tissues; IHC score values of these proteins were significantly higher in cancer tissues than in normal tissues. Bars indicate SD. ${ }^{*} \mathrm{P}<0.05$. 

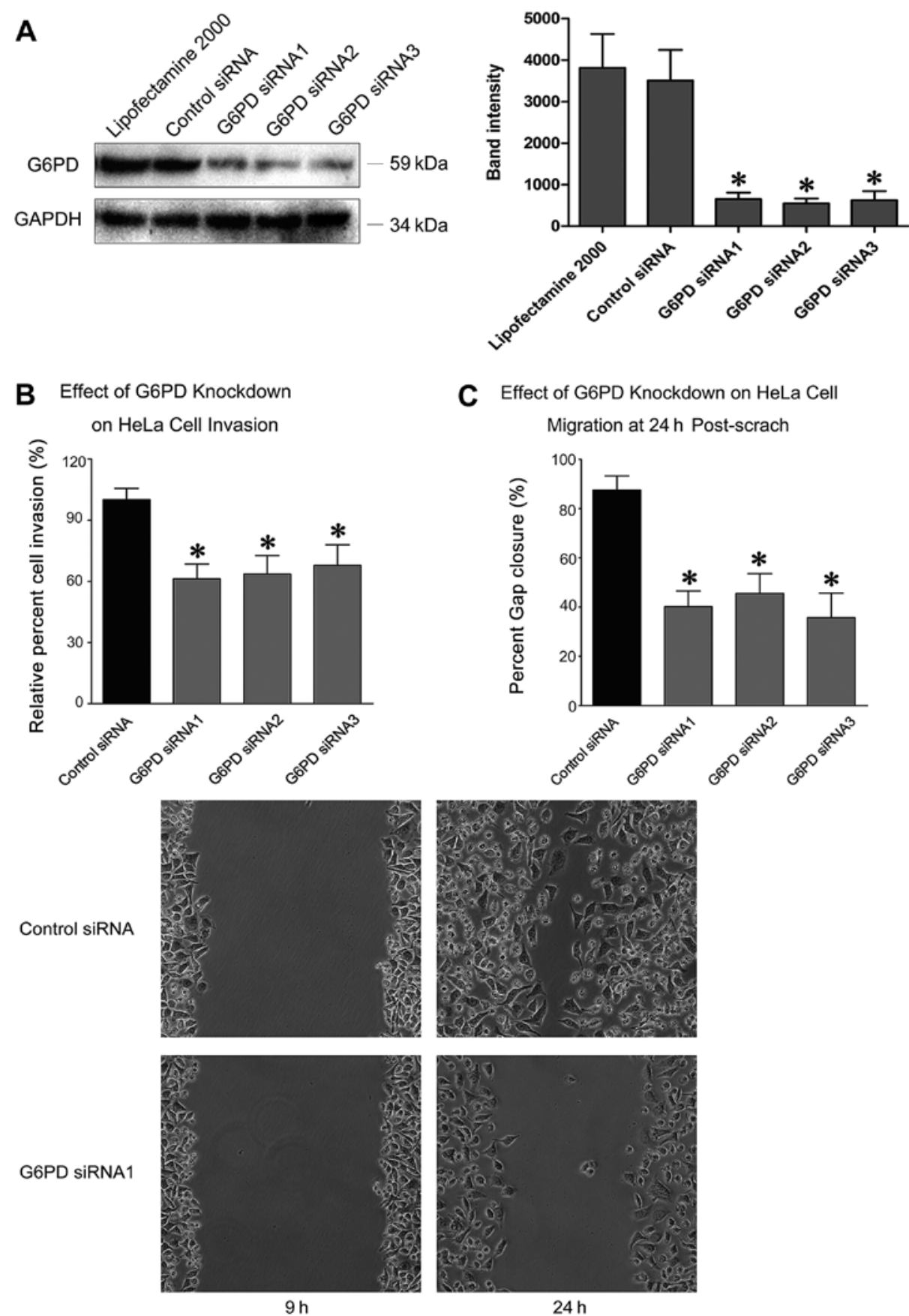

Figure 5. Functional studies of G6PD in HeLa cell migration and invasion. (A) G6PD protein levels in HeLa cells were dramatically reduced by transfection with G6PD-specific siRNAs. (B) G6PD silencing significantly inhibited the invasion properties of HeLa cells. (C) G6PD knockdown sharply reduced the ability of HeLa cells to close the gap introduced by a scratch wound. Bars indicate SD. " $\mathrm{P}<0.05$.

HeLa cells were decreased in vitro when G6PD expression was suppressed. Our results, together with findings of other groups, indicate that the suppression of G6PD expression may be beneficial in cervical cancer treatment.

Another markedly upregulated protein in cervical cancer found in the present study was STAT1, a member of STAT protein family. STAT1 was noted to be critical in controlling cell cycle progression and apoptosis. Hix et al (28) studied STAT1 effects by injecting mouse mammary carcinoma cell TM40D with/without ectopic STAT1 into the mammary fat pads of BALB/c mice and observed that the growth and aggressiveness of TM40D-STAT1 cells were dramatically enhanced, when compared to those of regular TM40D cells. Conversely, the growth of TM40D cells was significantly delayed when STAT1 was knocked down. Further studies demonstrated that STAT1 promoted breast cancer by increasing interleukin-13 (IL-13), transforming growth factor $\beta$ (TGF $\beta$ ) and tumor necrosis factor $\alpha$ (TNF $\alpha$ ). These factors could inhibit antitumor immune responses by recruiting and stimulating associated cells (29). In addition, Rajkumar et al (30) found that STAT1 rose in early cervical intraepithelial neoplasia (CIN1/2), dropped in CIN3/CIS, and was dramatically upregulated in invasive cervical carcinoma, demonstrating that STAT1 may harbor double roles in HPV infection and cervical tumori- 
genesis, specifically a protective role in the early phase and an oncogenic role in the invasive stages of the tumor. Further investigation is required to determine the roles of STAT1 in cervical tumorigenesis.

HSPB1 protein, also named heat shock protein 27 (Hsp27), belongs to the emerging 'survival protein' family (31-33). The expression of HSPB1 is involved in cell proliferation (34) and apoptosis (35) and is associated with poor prognosis in various tumors $(34,36)$. The inhibitor of HSPB1, OGX-427, a secondgeneration antisense oligonucleotide of HSPB1, downregulated the levels of HSPB1 and induced the apoptosis of cancer cells (37). This occurs via the disruption of the interaction of HSPB1 with cytochrome $c$ (cytc), procaspases-9 and -3 (35). Thus, the attenuation of the expression of HSPB1 gene was considered as a new therapeutic strategy. Moreover, McCollum et al (38) were able to successfully make prostate cancer cells more sensitive to the antitumor drug 17-allylamino-demethoxygeldanamycin by employing small-interfering RNA (siRNA) against HSPB1. Together, accumulating evidence supports the notion that upregulation of HSPB1 stimulates cancer cell proliferation, whereas its downregulation results in the inhibition of tumor progression.

ALDH3A1 proteins identified in the present study has been suggested involved in tumor progression, but not implicated in cervical cancer. The aldehyde dehydrogenase superfamily member ALDH3A1 belongs to the phase II group of drugmetabolizing enzymes and plays key roles in numerous biological processes, such as cell survival and growth (39). Upregulation of ALDH3A1 is frequent in tumor-derived cell lines and neoplastic tissues (40). By removing lipid peroxidation products, the overexpression of ALDH3A1 promoted the growth of rat hepatoma cells (41). In contrast, a reduced expression of ALDH3A1 inhibited the growth of cancer cells tested in lung tumor cell line A549 via activating peroxisome proliferator activated receptor $\gamma$ (PPAR $\gamma)$, which inhibits $\mathrm{NF}-\kappa \mathrm{B}$ activity (42). These findings are consistent with our observations made in cervical carcinoma and suggested that ALDH3A1 may be a promising candidate target of cervical cancer therapy.

In summary, we performed a non-targeted proteomics study to profile differentially expressed proteins in cervical cancer. The proteins uncovered, as well as studied, in the present study may serve as potential targets for cervical cancer research and treatment.

\section{Acknowledgements}

The present study was supported by grants from the National Basic Research Program of China (2010CB945103), the National Natural Science Foundation of China (81030013 and 81170002), the Science and Technology Project of Jiangsu Province (BL2014053), the Ph.D. Programs Foundation of Ministry of Education of China (20110091120032), and the Jiangsu Province Health Department of Medicine leading talent and innovation team project (LJ201109).

\section{References}

1. Parkin DM, Pisani P and Ferlay J: Estimates of the worldwide incidence of eighteen major cancers in 1985. Int J Cancer 54: 594-606, 1993.
2. Ferlay J, Shin HR, Bray F, Forman D, Mathers C and Parkin DM: Estimates of worldwide burden of cancer in 2008. Int J Cancer 127: 2893-2917, 2010.

3. Munoz N, Bosch FX, de Sanjose S, et al: The causal link between human papillomavirus and invasive cervical cancer: a population-based case-control study in Colombia and Spain. Int J Cancer 52: 743-749, 1992.

4. Schiffman MH, Bauer HM, Hoover RN, et al: Epidemiologic evidence showing that human papillomavirus infection causes most cervical intraepithelial neoplasia. J Natl Cancer Inst 85: 958-964, 1993.

5. Franco E, Syrjanen K, de Wolf C, et al: New developments in cervical cancer screening and prevention. Geneva, Switzerland, June 17-19 1996. Workshop. Cancer Epidemiol Biomarkers Prev 5: 853-856, 1996.

6. Franco EL: Measurement errors in epidemiological studies of human papillomavirus and cervical cancer. IARC Sci Publ 119: 181-197, 1992.

7. Sun W, Xing B, Sun Y, et al: Proteome analysis of hepatocellular carcinoma by two-dimensional difference gel electrophoresis: novel protein markers in hepatocellular carcinoma tissues. Mol Cell Proteomics 6: 1798-1808, 2006.

8. Bae SM, Lee CH, Cho YL, Nam KH, Kim YW, Kim CK, Han BD, Lee YJ, Chun HJ and Ahn WS: Two-dimensional gel analysis of protein expression profile in squamous cervical cancer patients. Gynecol Oncol 99: 26-35, 2005.

9. Bae SM, Min HJ, Ding GH, et al: Protein expression profile using two-dimensional gel analysis in squamous cervical cancer patients. Cancer Res Treat 38: 99-107, 2006.

10. Abdul-Rahman PS, Lim BK and Hashim OH: Expression of high-abundance proteins in sera of patients with endometrial and cervical cancers: analysis using 2-DE with silver staining and lectin detection methods. Electrophoresis 28: 1989-1996, 2007.

11. Görg A, Obermaier C, Boguth G, Harder A, Scheibe B, Wildgruber R and Weiss W: The current state of two-dimensional electrophoresis with immobilized $\mathrm{pH}$ gradients. Electrophoresis 21: 1037-1053, 2007

12. Fu C, Wu C, Liu T, Ago T, Zhai P, Sadoshima J and Li H: Elucidation of thioredoxin target protein networks in mouse. Mol Cell Proteomics 8: 1674-1687, 2009.

13. Rangiah K, Tippornwong M, Sangar V, Austin D, Tétreault MP, Rustgi AK, Blair IA and Yu K: Differential secreted proteome approach in murine model for candidate biomarker discovery in colon cancer. J Proteome Res 8: 5153-5164, 2009.

14. Ross PL, Huang YN, Marchese JN, et al: Multiplexed protein quantization in Saccharomyces cerevisiae using amine-reactive isobaric tagging reagents. Mol Cell Proteomics 3: 1154-1169, 2004.

15. Yang Y, Toy W, Choong LY, Hou P, Ashktorab H, Smoot DT, Yeoh KG and Lim YP: Discovery of SLC3A2 cell membrane protein as a potential gastric cancer biomarker: implications in molecular imaging. J Proteome Res 1: 5736-5747, 2012.

16. Chen Y, Choong LY, Lin Q, et al: Differential expression of novel tyrosine kinase substrates during breast cancer development. Mol Cell Proteomics 6: 2072-2087, 2007.

17. Chong PK, Lee H, Zhou J, Liu SC, Loh MC, So JB, Lim KH, Yeoh KG and Lim YP: Reduced plasma APOA1 level is associated with gastric tumor growth in MKN45 mouse xenograft model. J Proteomics 73: 1632-1640, 2010.

18. Fujii K, Kondo T, Yokoo H, Yamada T, Iwatsuki $\mathrm{K}$ and Hirohashi S: Proteomic study of human hepatocellular carcinoma using two-dimensional difference gel electrophoresis with saturation cysteine dye. J Proteomics 5: 1411-1422, 2005.

19. Gan CS, Chong PK, Pham TK and Wright PC: Technical, experimental, and biological variations in isobaric tags for relative and absolute quantitation (iTRAQ). J Proteome Res 6: 821-827, 2007.

20. Pandolfi PP, Sonati F, Rivi R, Mason P, Grosveld F and Luzzatto L: Targeted disruption of the housekeeping gene encoding glucose 6-phosphate dehydrogenase (G6PD): G6PD is dispensable for pentose synthesis but essential for defense against oxidative stress. EMBO J 14: 5209-5215, 1995.

21. Hu T, Zhang C, Tang Q, Su Y, Li B, Chen L, Zhang Z, Cai T and Zhu Y: Variant G6PD levels promote tumor cell proliferation or apoptosis via the STAT3/5 pathway in the human melanoma xenograft mouse model. BMC Cancer 13: 251, 2013.

22. Jiang P, Du W and Yang X: A critical role of glucose-6-phosphate dehydrogenase in TAp73-mediated cell proliferation. Cell Cycle 12: 3720-3726, 2013 
23. Lin HR, Wu CC, Wu YH, Hsu CW, Cheng ML and Chiu DT: Proteome-wide dysregulation by glucose-6-phosphate dehydrogenase (G6PD) reveals a novel protective role for G6PD in aflatoxin B(1)-mediated cytotoxicity. J Proteome Res 12: 3434-3448, 2013.

24. DeBerardinis RJ, Lum JJ, Hatzivassiliou G and Thompson CB: The biology of cancer: metabolic reprogramming fuels cell growth and proliferation. Cell Metab 7: 11-20, 2008.

25. Vander Heiden MG, Cantley LC and Thompson CB: Understanding the Warburg effect: the metabolic requirements of cell proliferation. Science 324: 1029-1033, 2009.

26. Jiang P, Du W, Wang X, Mancuso A, Gao X, Wu M and Yang X: p53 regulates biosynthesis through direct inactivation of glucose-6-phosphate dehydrogenase. Nat Cell Biol 13: 310-316, 2011.

27. Berg J, Tymoczko J and Stryer L: Biochemistry. 6th edition. WH Freeman \& Co, New York, NY, pp577-589, 2006.

28. Hix LM, Karavitis J, Khan MW, Shi YH, Khazaie K and Zhang M: Tumor STAT1 transcription factor activity enhances breast tumor growth and immune suppression mediated by myeloid-derived suppressor cells. J Biol Chem 288: 11676-11688, 2013.

29. Zhang M: Novel function of STAT1 in breast cancer. Oncoimmunology 2: e25125, 2013.

30. Rajkumar T, Sabitha K, Vijayalakshmi N, Shirley S, Bose MV, Gopal $\mathrm{G}$ and Selvaluxmy G: Identification and validation of genes involved in cervical tumourigenesis. BMC Cancer 11: 80 2011.

31. Altieri DC: Survivin, versatile modulation of cell division and apoptosis in cancer. Oncogene 22: 8581-8589, 2003.

32. Ciocca DR and Calderwood SK: Heat shock proteins in cancer: diagnostic, prognostic, predictive, and treatment implications. Cell Stress Chaperones 10: 86-103, 2005.

33. Cory S, Huang DC and Adams JM: The Bcl-2 family: roles in cell survival and oncogenesis. Oncogene 22: 8590-8607, 2003.
34. Romanucci M, Marinelli A, Sarli G and Della Salda L: Heat shock protein expression in canine malignant mammary tumours. BMC Cancer 6: 171, 2006.

35. Bruey JM, Ducasse C, Bonniaud P, et al: Hsp27 negatively regulates cell death by interacting with cytochrome. Nat Cell Biol 2: 645-652, 2000

36. Cornford PA, Dodson AR, Parsons KF, Desmond AD, Woolfenden A, Fordham M, Neoptolemos JP, Ke Y and Foster CS: Heat shock protein expression independently predicts clinical outcome in prostate cancer. Cancer Res 60: 7099-7105, 2000.

37. Cohen M, Dromard M and Petignat P: Heat shock proteins in ovarian cancer: a potential target for therapy. Gynecol Oncol 119: 164-166, 2010.

38. McCollum AK, Teneyck CJ, Sauer BM, Toft DO and Erlichman C: Up-regulation of heat shock protein 27 induces resistance to 17-allylamino-demethoxygeldanamycin through a glutathionemediated mechanism. Cancer Res 66: 10967-10975, 2006.

39. Vasiliou V and Nebert DW: Analysis and update of the human aldehyde dehydrogenase (ALDH) gene family. Hum Genomics 2: 138-143, 2005.

40. Vasiliou V, Pappa A and Estey T: Role of human aldehyde dehydrogenases in endobiotic and xenobiotic metabolism. Drug Metab Rev 36: 279-299, 2004.

41. Canuto RA, Muzio G, Ferro M, Maggiora M, Federa R, Bassi AM, Lindahl R and Dianzani MU: Inhibition of class-3 aldehyde dehydrogenase and cell growth by restored lipid peroxidation in hepatoma cell lines. Free Radic Biol Med 26: 333-340, 1999.

42. Muzio G, Trombetta A, Maggiora M, Martinasso G, Vasiliou V, Lassen N and Canuto RA: Arachidonic acid suppresses growth of human lung tumor A549 cells through down-regulation of ALDH3A1 expression. Free Radic Biol Med 40: 1929-1938, 2006. 